\title{
EVOLUTION OPERATORS FOR QUANTUM CHAINS
}

\author{
S. SERGEEV
}

\begin{abstract}
Discrete-time evolution operators in integrable quantum lattice models are sometimes more fundamental objects then Hamiltonians. In this paper we study an evolution operator for the one-dimensional integrable $q$-deformed Bose gas with XXZ-type impurities and find its spectrum. Evolution operators give a new interpretation of known integrable systems, for instance our system describes apparently a simplest laser with a clear resonance peak in the spectrum.
\end{abstract}

Existence of a complete set of commuting operators is the basic principle of quantum integrability. However, often the commuting set does not provide a distinguished operator deserving the title of the Hamiltonian for a physical system. In particular, this is the common feature of quantum models obtained by quantization of classical equations of motion in wholly discrete space-time. Equations of motion define a discrete time translations for classical variables, a map $A(\tau, \sigma) \rightarrow A(\tau+1, \sigma)$. Corresponding translation for quantum observables is produced by an evolution operator,

$$
\mathbf{A}(\tau+1, \sigma)=\mathbf{U A}(\tau, \sigma) \mathbf{U}^{\dagger}, \quad \mathbf{U U}^{\dagger}=1
$$

If the time unity interval coincides with the unity spacing, then there is no a small parameter expansion of $\mathbf{U}$ defining a lattice Hamiltonian. The evolution operator becomes the main object of the lattice quantum mechanics.

Discrete-time evolution operators for quantum chains were considered in many papers. For instance, the study of the spectrum of evolution operator was in the focus of the quantum Liouville theory [1]. In this paper we discuss another example of quantum evolution system: the one-dimensional $q$-deformed Bose gas [2] with $X X Z$-type impurities. The simple test "what evolution operator is doing in the space of states" will give a new view on well known models.

Quantum mechanics begins with the algebra of observables. In our case the local algebra of observables is the $q$-oscillator $\left[3\right.$ with generators $\mathbf{B}, \mathbf{B}^{\dagger}, \mathbf{N}$ :

$$
\mathbf{B B}^{\dagger}=1-q^{2 \mathbf{N}+2}, \quad \mathbf{B}^{\dagger} \mathbf{B}=1-q^{2 \mathbf{N}}, \quad 0<q<1 .
$$

1991 Mathematics Subject Classification. 37K15.

Key words and phrases. One-Dimensional Bose Gas, Spin Chains, Quantum Integrable Systems, Bethe Ansatz, Evolution Operators.

This work was supported by the Australian Research Council. 
Below we use the Fock space representation of (2), the Fock vacuum is defined by $\mathbf{B}|0\rangle=0$. The $q$-oscillators are the algebra of observables for $q$-deformed Bose gas. In addition, it is very convenient to use the $q$-oscillator representation for $X X Z$ model [4, [5]. In this paper we consider the combined chain of three types of $q$-oscillators,

$$
\left\{\mathbf{B}_{j, n}, \mathbf{B}_{j, n}^{\dagger}, \mathbf{N}_{j, n}\right\} \quad: \quad j=1,2,3, \quad n=0,1, \ldots, N-1,
$$

where index ${ }_{3, n}$ stands for the $q$-Bose gas, indices ${ }_{1, n}$ and ${ }_{2, n}$ stand for oscillator representation of $X X Z$ model in $n^{\text {th }}$ site of the combined chain. The quantum Lax operators are

$$
M_{n}(u)=\left(\begin{array}{cc}
-q^{\mathbf{N}_{3, n}} & u \mathbf{B}^{\dagger}{ }_{3, n} \\
q^{-1} \mathbf{B}_{3, n} & u q^{\mathbf{N}_{3, n}}
\end{array}\right)
$$

for the $q$-Bose gas (condition $q<1$ is equivalent to the attractive potential), and

$$
L_{n}(u)=\left(\begin{array}{cc}
u q^{\mathbf{N}_{1, n}}+\mathrm{e}^{\mathrm{i} \varepsilon} q^{\mathbf{N}_{2, n}} & -u \mathrm{e}^{\mathrm{i} \varepsilon} \mathbf{B}_{1, n} \mathbf{B}^{\dagger}{ }_{2, n} \\
q^{-1} \mathbf{B}_{2, n} \mathbf{B}^{\dagger}{ }_{1, n} & u q^{\mathbf{N}_{2, n}}+\mathrm{e}^{\mathrm{i} \varepsilon} q^{\mathbf{N}_{1, n}}
\end{array}\right)
$$

for the $X X Z$ model. $L$-operator (5) has the center

$$
\mathbf{s}_{n}=\frac{1}{2}\left(\mathbf{N}_{1, n}+\mathbf{N}_{2, n}\right)
$$

with the half-integer spectrum, matrix (5) on the subspace $\mathbf{s}_{n}=s_{n}$ is identical to the usual spin- $s_{n}$ $L$-operator for $\mathcal{U}_{q}\left(\widehat{s l}_{2}\right)$. Note, on the subspace $\mathbf{s}_{n}=0$ operator (5) is just unity matrix, $\left.L_{n}(u)\right|_{\mathbf{s}_{n}=0}=$ $\left(u+\mathrm{e}^{\mathrm{i} \varepsilon}\right) \mathbb{1}$. Extra parameter $\varepsilon$ in (5) is equivalent to an exponential shift of the spectral parameter $u$.

The transfer matrix of the model is defined by

$$
t(u)=\operatorname{Trace}\left(M_{0}(u) L_{0}(u) M_{1}(u) L_{1}(u) M_{2}(u) L_{2}(u) \cdots M_{N-1}(u) L_{N-1}(u)\right)
$$

Complete set of commutative operators is given by the decomposition of $t(u)$ and by the set of charges $\mathbf{s}_{n}$ (6). Subjects of our interest are sub-sectors of the extended Hilbert space corresponding to specified eigenvalues of $\mathbf{s}_{n}$. In particular, if all $\mathbf{s}_{n}=0$, this is just the pure one-dimensional $q$-Bose gas with attractive potential. If $\mathbf{s}_{0}=1 / 2$ and all other $\mathbf{s}_{n}=0$, this is the Bose gas with the single impurity. If some of $\mathbf{s}_{n}=1 / 2$ and all the other $\mathbf{s}_{n}=0$ (our final case), this is the $q$-Bose gas with some density of impurities.

Our main object, the discrete-time evolution operator, is defined by

$$
\mathbf{U} M_{n}(u) L_{n}(u)=L_{n}(u) M_{n+1}(u) \mathbf{U} .
$$


Evidently, transfer matrix (7) commutes with the evolution operator and therefore gives the integrals of motion. One can rewrite (8) as the map:

$$
\begin{aligned}
& \mathbf{U} q^{\mathbf{N}_{2, n}} \mathbf{B}^{\dagger}{ }_{1, n} \mathbf{U}^{-1}=q^{\mathbf{N}_{3, n}} \mathbf{B}_{1, n}^{\dagger}-\mathrm{e}^{\mathrm{i} \varepsilon} q^{\mathbf{N}_{1, n}} \mathbf{B}_{2, n}^{\dagger} \mathbf{B}_{3, n}, \\
& \mathbf{U ~ B ~}^{\dagger}{ }_{2, n} \mathbf{U}^{-1}=\mathbf{B}^{\dagger}{ }_{1, n} \mathbf{B}^{\dagger}{ }_{3, n}+q \mathrm{e}^{\mathrm{i} \varepsilon} q^{\mathbf{N}_{1, n}+\mathbf{N}_{3, n}} \mathbf{B}_{2, n}^{\dagger}, \\
& \mathbf{U} q^{\mathbf{N}_{2, n+1}} \mathbf{B}_{3, n}^{\dagger} \mathbf{U}^{-1}=q^{\mathbf{N}_{1, n+1}} \mathbf{B}_{3, n+1}^{\dagger}-\mathrm{e}^{\mathrm{i} \varepsilon} q^{\mathbf{N}_{3, n+1}} \mathbf{B}_{1, n+1} \mathbf{B}^{\dagger}{ }_{2, n+1} .
\end{aligned}
$$

Corresponding relations for $\mathbf{U B}_{j, n} \mathbf{U}^{-1}$ are the conjugation of (9): $\mathbf{U}$ is the unitary operator if $q$ and $\varepsilon$ are real. Equations (9) provide in addition $\mathbf{U s}_{n}=\mathbf{s}_{n} \mathbf{U}$ and $\mathbf{U K}=\mathbf{K U}$ where

$$
\mathbf{K}=\sum_{n}\left(\mathbf{N}_{2, n}+\mathbf{N}_{3, n}\right) \text {. }
$$

Note, the map (9) is exactly the tetrahedral map from [5. All two-dimensional models have hidden three-dimensional structure.

Take up now the interpretation of the evolution operator. In what follows, we use

$$
q=\mathrm{e}^{-\gamma}
$$

We start with the lowest occupation numbers over the total Fock vacuum $|0\rangle$. Let $\mathbf{U}|0\rangle=|0\rangle$, and we consider next the subspace of the Hilbert space with $\mathbf{s}_{0}=\mathbf{K}=1$. Let us see, what the evolution operator makes with the state

$$
\left|\psi_{1}\right\rangle=\mathbf{B}_{2,0}^{\dagger}|0\rangle
$$

Applying (9) once, we get

$$
\mathbf{U}\left|\psi_{1}\right\rangle=\mathrm{e}^{\mathrm{i} \varepsilon-\gamma}\left|\psi_{1}\right\rangle+\left|\psi_{0}\right\rangle \otimes\left|b_{0}\right\rangle
$$

where we use the notation

$$
\left|\psi_{0}\right\rangle \otimes\left|b_{k}\right\rangle=\mathbf{B}_{1,0}^{\dagger} \mathbf{B}_{3, k}^{\dagger}|0\rangle .
$$

What is happening after $\tau$ steps of discrete evolution:

$$
\mathbf{U}^{\tau}\left|\psi_{1}\right\rangle=\mathrm{e}^{(\mathrm{i} \varepsilon-\gamma) \tau}\left|\psi_{1}\right\rangle+\mathrm{e}^{-(\mathrm{i} \varepsilon-\gamma)}\left|\psi_{0}\right\rangle \otimes\left(\sum_{k=0}^{\tau-1} \mathrm{e}^{(\mathrm{i} \varepsilon-\gamma)(\tau-k)}\left|b_{k}\right\rangle\right) .
$$

Suppose, $\tau$ is big enough, but

$$
\gamma \tau \ll 1, \quad \varepsilon \tau \simeq \text { some integer multiple of } 2 \pi .
$$

Then equation (15) describes the decay the quasi-stable state $\left|\psi_{1}\right\rangle$ with the energy $\varepsilon$ and width $\gamma$. The second term in (15) is a state with the bosonic wave function $\Psi(\tau, k)=\mathrm{e}^{(i \varepsilon-\gamma)(\tau-k)}-$ the speed-of-light 
right-moving wave radiated by excited state. Due to (16), the oscillating term in the wave function dominates, and therefore the wave package in the right hand side of (15) is the state with the average energy and momentum $\varepsilon$. Note, we may talk about the energy of the radiated state only if $\tau$ in (15) is big enough, this exactly corresponds to the Heisenberg uncertainty principle. The one-step distance of the lattice may be considered as the ultra-microscopic scale of the space-time, while big $\tau$ in (15) corresponds to a microscopic scale.

Thus, the discrete-time evolution operator provides an alternative terminology. We will call site $n$ of the spin chain with $s_{n}=1 / 2$ as the atom with two energy states: the ground state $\left|\psi_{0}\right\rangle$ with zero energy and the excited state $\left|\psi_{1}\right\rangle$ with the energy $\varepsilon$ and width $\gamma$. The regime for $\gamma$ and $\varepsilon$ follows from (16):

$$
\gamma \ll \varepsilon \ll 2 \pi
$$

The spin chain sites with $s_{n}=0$ do not provide any effect, these sites are just empty. The Bose field $\mathbf{B}_{3, n}^{\dagger}$ is identified with the electro-magnetic field, the radiated state in (15) is the photon. The whole system is evidently a kind of chiral laser (photons are radiated to the right only). Note, the impurities destroy the translation invariance of the system. Scattered and radiated photons may have momenta, but the atoms are rigidly fixed and the solid media takes up the recoil momenta of photons.

Consider next eigenstates and eigenvalues of the evolution operator. We consider the periodical boundary conditions - a "toroidal chiral laser". The eigenstates have the structure of the superposition of stationary bosonic waves scattering on the atoms (the coordinate Bethe Ansatz). For instance, the one-boson and one-atom eigenstate $\left|\Psi_{p}\right\rangle$, such that $\mathbf{U}\left|\Psi_{p}\right\rangle=\mathrm{e}^{\mathrm{i} p}\left|\Psi_{p}\right\rangle$, is

$$
\left|\Psi_{p}\right\rangle=-\frac{\mathrm{e}^{\mathrm{i}(p+\varepsilon)}\left(1-q^{2}\right)}{q \mathrm{e}^{\mathrm{i} p}-\mathrm{e}^{\mathrm{i} \varepsilon}} \mathbf{B}_{2,0}^{\dagger}|0\rangle+\mathbf{B}_{1,0}^{\dagger} \mathbf{B}_{p}^{\dagger}|0\rangle,
$$

where

$$
\mathbf{B}_{p}^{\dagger}=\sum \mathrm{e}^{-\mathrm{i} p k} \mathbf{B}_{3, k}^{\dagger}
$$

is the creation operator of the photon. The quantization equation for the momenta of one-photon states follows from the periodical boundary conditions,

$$
\mathrm{e}^{\mathrm{i} N p} \frac{\mathrm{e}^{\mathrm{i} p}-q \mathrm{e}^{\mathrm{i} \varepsilon}}{q \mathrm{e}^{\mathrm{i} p}-\mathrm{e}^{\mathrm{i} \varepsilon}}=1
$$

Since the evolution operator describes only the right-moving photons with dispersion relation $E=p$, the momentum $p$ in (1920) must be positive. 
In general, the spectrum of $\mathbf{U}$ is given by

$$
\mathbf{U}=\prod_{i=1}^{K} u_{i}=\exp \left\{\mathrm{i} \sum_{i=1}^{K} p_{i}\right\}
$$

where $u_{i}=\mathrm{e}^{\mathrm{i} p_{i}}$ are the roots of the Bethe Ansatz equations

$$
u_{i}^{N} \prod_{n} \frac{u_{i}-q^{2 s_{n}} \mathrm{e}^{\mathrm{i} \varepsilon}}{q^{2 s_{n}} u_{i}-\mathrm{e}^{\mathrm{i} \varepsilon}}=\prod_{j \neq i} \frac{q^{-1} u_{i}-q u_{j}}{q u_{i}-q^{-1} u_{j}}, \quad i, j=1, \ldots, K .
$$

Here $N$ is the length of the chain, periodical boundary conditions are taken into account, and $K$ is the eigenvalue of (10) (the number of photons in the laser). In general, $s_{n}$ are arbitrary eigenvalues of $\mathbf{s}_{n}$, but here we consider $s_{n}=0,1 / 2$ only. Equations (22) are literally the Bethe Ansatz equations for transfer matrix (7); relation (21) is the main analytical result of this paper.

The eigenstates corresponding to (22) may be interpreted in terms of the photon creation operators in momentum space (19). If all $p_{i}$ are real and different, the photonic counterpart of the eigenstate is a slightly modified state $\mathbf{B}^{\dagger}{ }_{p_{1}} \mathbf{B}^{\dagger} p_{2} \ldots \mathbf{B}^{\dagger} p_{K}|0\rangle$. In addition to real $p_{i}$, the Bethe Ansatz equations (22) have solutions with complex $p_{i}$ (strings of the Bethe Ansatz equations), for instance $p_{1} \simeq p+\mathrm{i} \gamma$ and $p_{2} \simeq p-\mathrm{i} \gamma$. The bosonic counterpart of such eigenstate is $\sum_{k, k^{\prime}} \mathrm{e}^{-\mathrm{i} p\left(k+k^{\prime}\right)-\gamma\left|k-k^{\prime}\right|} \mathbf{B}_{3, k}^{\dagger} \mathbf{B}_{3, k^{\prime}}^{\dagger}|0\rangle$. This is the bound state for big $\gamma$, but in our regime (17) it may be understood as properly $\gamma$-regularized state $\mathbf{B}_{p}^{\dagger_{p}^{2}}|0\rangle$. Analogously, the highest bound states correspond to the highest occupation numbers $\mathbf{B}_{p}^{\dagger}|0\rangle$. The important point is that we have to solve the Bethe Ansatz equations implying the right-moving condition $\operatorname{Re}\left(p_{i}\right)>0$.

Turn finally to the thermodynamical limit $N, K \rightarrow \infty$. Let the number of two-states atoms be $N^{\prime}<N$, their density $n=N^{\prime} / N$ is not zero at the limit. Structure of the infrared part of spectrum is the subject of separate investigation. The lowest energy levels correspond to Bethe Ansatz strings, the $\gamma$-regularization solves apparently the infrared catastrophe problem.

In this paper we consider the "optical wave band" $p \sim \varepsilon$. Assume that the Bethe roots $u_{i}=\mathrm{e}^{\mathrm{i} p_{i}}$, $\operatorname{Im}\left(p_{i}\right)=0$, form a dense distribution $\varrho\left(p_{i}\right)^{-1}=N\left(p_{i+1}-p_{i}\right)$ near $p_{i} \sim \varepsilon$. Assume in addition that we can neglect in this region densities of photons with higher occupation numbers (complex $\left.p_{i}\right)$. The standard Bethe Ansatz technique provides the Hulthen integral equation for the density $\varrho(p)$ :

$$
1-2 \pi \varrho(p)=\int d p^{\prime} \varrho\left(p^{\prime}\right) \frac{d}{d p} S\left(p, p^{\prime}\right)+n \frac{d}{d p} F(p),
$$

where

$$
\mathrm{e}^{\mathrm{i} S\left(p, p^{\prime}\right)}=\frac{\mathrm{e}^{\mathrm{i} p+\gamma}-\mathrm{e}^{\mathrm{i} p^{\prime}-\gamma}}{\mathrm{e}^{\mathrm{i} p-\gamma}-\mathrm{e}^{\mathrm{i} p^{\prime}+\gamma}}, \quad \mathrm{e}^{\mathrm{i} F(p)}=\frac{\mathrm{e}^{\mathrm{i} p-\gamma}-\mathrm{e}^{\mathrm{i} \varepsilon}}{\mathrm{e}^{\mathrm{i} p}-\mathrm{e}^{\mathrm{i} \varepsilon-\gamma}} .
$$


Solving (23) in the optical wave band, we neglect the contribution of the radio band since $\frac{d}{d p} S\left(p, p^{\prime}\right) \rightarrow$ 0 when $\left|p-p^{\prime}\right| \gg \gamma$. The solution is

$$
\varrho(p) \simeq(4 \pi)^{-1}+\frac{n}{4 \gamma} \frac{1}{\cosh \frac{\pi(p-\varepsilon)}{2 \gamma}},
$$

what is evidently the laser resonance peak over the white noise. Accounting of radio band and complex $p_{i}$ may modify the white noise term. The resonance term has exactly the $X X X$-spin chain structure, so that the number of resonant photons in the stationary state is half of the number of atoms. Additionally pumped energy does not increase the density of resonant photons, it dissipates into the white noise.

Acknowledgements. I would like to thank M. Bortz, J. de Gier and V. Mangazeev for fruitful discussions.

\section{REFERENCES}

[1] L. D. Faddeev, R. M. Kashaev and A. Yu. Volkov, "Strongly coupled quantum discrete Liouville theory. I. Algebraic approach and duality", Comm. Math. Phys. 219 (2001) 199-219.

L. D. Faddeev and R. M. Kashaev, "Strongly coupled quantum discrete Liouville theory. II. Geometric interpretation of the evolution operator", J. Phys. A 35 (2002) 4043-4048.

[2] N. M. Bogoliubov and R. K. Bullough, "A q-deformed completely integrable Bose gas model", J. Phys. A 25 (1992) $4057-4071$.

N. M. Bogoliubov, A. G. Izergin and N. A. Kitanine, "Correlation functions for a strongly correlated boson system", Nuclear Phys. B 516 (1998) 501-528.

[3] E. V. Damaskinsky and P. P. Kulish, "Deformed oscillators and their applications", J. Soviet Math. 62 (1992) 2963-2986

[4] A. J. Macfarlane, "On $q$-analogues of the quantum harmonic oscillator and the quantum group $S U(2)_{q}$ ", J. Phys. A: Math. Gen. 22 (1989) 4581-4588

L. C. Biedenharn, "The quantum group $S U_{q}(2)$ and a $q$-analogue of boson operators", J. Phys. A: Math. Gen. 22 (1989) L873-L878

[5] V. V. Bazhanov and S. M. Sergeev, "Zamolodchikov's tetrahedron Equation and Hidden Structure of Quantum Groups", J. Phys. A: Math. Gen. 39 (2006) 3295-3310

Department of Theoretical Physics (Research School of Physical Sciences and Engineering) \& Mathematical Science Institute, Canberra ACT 0200, Australia

E-mail address: sergey.sergeev@anu.edu.au 